04

\title{
Формирование двух импульсов тока пучка убегающих электронов
}

\author{
(ㄷ Д.В. Белоплотов, В.Ф. Тарасенко, Д.А. Сорокин, В.А. Шкляев \\ Институт сильноточной электроники СО РАН, \\ 634055 Томск, Россия \\ e-mail: VFT@loi.hcei.tsc.ru
}

Поступило в Редакцию 14 октября 2020 г.

В окончательной редакции 17 ноября 2020г.

Принято к публикации 19 ноября 2020 г.

Исследованы условия формирования двух импульсов тока пучка убегающих электронов при пробое промежутков острие-плоскость и трубка-плоскость в воздухе, азоте и гелии повышенного давления. Экспериментально показано, что в зависимости от давления и сорта газа при различной длительности фронта импульса напряжения амплитудой в десятки киловольт наблюдаются три режима генерации пучка убегающих электронов. В первом режиме во время старта стримера с катода происходит генерация одиночного импульса тока пучка при максимальном напряжении на промежутке. Его длительность за анодом составляет 100 ps. Два импульса тока пучка наблюдаются во втором режиме при уменьшении давления. Момент времени генерации первого импульса сохраняется, а второй регистрируется после замыкания промежутка стримером (первой волной ионизации). Энергия электронов во втором импульсе существенно меньше, чем в первом, но длительность и амплитуда тока пучка в оптимальных условиях больше. В третьем режиме, который реализуется при более низких давлениях по сравнению со вторым режимом, ток пучка после первого импульса продолжается без паузы в квазистационарной стадии и имеет длительность сотни пикосекунд.

Ключевые слова: генерация убегающих электронов, сверхкороткий лавинный электронный пучок, второй ипульс тока пучка.

DOI: 10.21883/JTF.2021.04.50621.292-20

\section{Введение}

Генерация убегающих электронов с высокой энергией при импульсных разрядах в газах повышенного давления является важным физическим явлением, которое продолжает изучаться в настоящее время. Возможность получения убегающих электронов или(и) тормозного рентгеновского излучения в газах повышенного давления следует из теоретических работ, а их наличие зарегистрировано в лабораторных и атмосферных разрядах, см., например, [1-14]. В воздухе атмосферного давления пучки убегающих электронов за тонкой анодной фольгой фиксировались шунтом или коллектором в четырех научных группах $[7,9,10,14]$. Теоретические модели, см., например, [15-21], объясняют ряд важных тенденций при генерации пучков электронов. Однако полученные параметры и реализованные режимы генерации пучков убегающих электронов в различных экспериментальных работах $[7,9,10,14]$ существенно отличаются. Предлагаются различные механизмы генерации убегающих электронов. Например, за счет ,пространственного заряда ионов“ [22], „эффекта поляризационного самоускорения электронов“" [23], нагрева газа в микроканалах, который „обеспечивает рост отношения напряженности электрического поля к концентрации газа в микроканалах до значений, достаточных для генерации в них пучков высокоэнергетичных электронов и тормозного рентгеновского излучения“" [24], и другие [7-21].

Один из вопросов, который требует изучения это условия и механизм генерации двух импульсов тока пучка убегающих электронов, регистрируемых при повышенных давлениях, с одним импульсом напряжения. Впервые этот режим был получен при напряжении $\approx 25 \mathrm{kV}$ за счет вариации давления азота и гелия $[25,26]$. Импульс напряжения имел фронт $5 \mathrm{~ns}$ и длительность 20-90 ns. Катод был сферическим или трубчатым, а анод - плоским из металлической сетки. В азоте два импульса наблюдались при давлении $p<60$ Torr, а в гелии < 300 Torr. Первый импульс тока пучка генерировался на плоской части импульса напряжения. Данный импульс соответствовал режиму генерации сверхкороткого лавинного электронного пучка (СЛЭП) [9,12]. Уменьшение напряжения после генерации первого импульса в $[25,26]$ составляло не более $30 \%$. Длительность второго импульса тока пучка (ВИТП) была существенно больше ( $1 \mathrm{~ns})$ и зависела от давления и сорта газа. Временной интервал между максимумами двух импульсов тока пучка в этих условиях равнялся $\sim 1.5 \mathrm{~ns}$ и увеличивался с увеличением межэлектродного зазора. При увеличении давления (в гелии $\geq 300$ Torr, в азоте $\geq 60$ Torr), ВИТП исчезал и регистрировался только СЛЭП с длительностью на полувысоте $\approx 200$ ps. Амплитуда ВИТП в оптимальных условиях превышала амплитуду СЛЭП. Оценки энергии электронов по их ослаблению фильтрами показали, что в СЛЭП основная часть электронов имеет максимальную энергию $\sim 10 \mathrm{keV}$ при разряде в гелии и $\sim 12 \mathrm{keV}$ при разряде в азоте. Энергия электронов второго импульса при повышенном давлении не превышала $3 \mathrm{keV}$. 
Подобные результаты были получены в работе [27]. Использовались импульсы напряжения с амплитудой до $35 \mathrm{kV}$ и длительностью фронта $200 \mathrm{~ns}$ и катод из швейной иглы. Длительность второго импульса на полувысоте при давлении воздуха 95 Torr более чем в два раза превышала длительность первого и составила на полувысоте 300 ps. C увеличением давления второй импульс переставал регистрироваться. В работах $[25,26]$ предполагалось, что формирование второго импульса обусловлено второй волной ионизации, которая двигалась от катода с малым радиусом кривизны к аноду. Отметим, что при низких давлениях и пробое длинных трубок вторая волна ионизации могла возникать за счет взрывной эмиссии электронов, и в области ее фронта регистрировалось рентгеновское излучение [28].

Подробный анализ различных режимов генерации нескольких импульсов тока пучка убегающих электронов был проведен в [29]. Было отмечено, что увеличение амплитуды импульса напряжения генератора до $160 \mathrm{kV}$ позволило получить два импульса тока пучка при атмосферном давлении азота. С катодом, который состоял из четырех коаксиально расположенных трубок из фольги толщиной $50 \mu \mathrm{m}$ и имел общую длину кромки с малым радиусом кривизны $45 \mathrm{~cm}$, при межэлектродном зазоpe $4.5 \mathrm{~cm}$ и генерации СЛЭП напряжение на промежутке существенно не изменялось. При этом ВИТП наблюдается на фронте импульса тока разряда в стадии формирования завершенного пробоя промежутка. Два импульса тока пучка во время одного импульса напряжения с длительностью фронта $\approx 0.5 \mathrm{~ns}$ и амплитудой $\approx 250 \mathrm{kV}$ были получены также при давлении азота $\sim 2700$ Torr. СЛЭП и ВИТП регистрировались в условиях уменьшения параметра $E / p(E-$ напряженность электрического поля, $p$ - давление газа) за счет увеличения давления азота. Напряжение на промежутке после окончания СЛЭП уменьшалось на $\approx 30 \%$ и тем не менее его было достаточно для формирования второго импульса. Амплитуда ВИТП при выводе через сетку была больше, чем у СЛЭП. Задержка между максимумами двух импульсов с увеличением напряжения уменьшилась до $\sim 150$ ps. Электроны ВИТП имели энергию $<40 \mathrm{keV}$.

Кроме того, в работе [29] режим с двумя и более импульсами тока пучка субнаносекундной длительности был зарегистрирован в условиях высоких значений параметра $E / p$, которые достигались за счет уменьшения давления в газовом диоде. В гелии, водороде, неоне, азоте и воздухе при давлениях единицы-десятки Torr, aмплитуде наносекундного импульса напряжения $\approx 250 \mathrm{kV}$ и установке после анодной фольги металлической диафрагмы с миллиметровым отверстием был реализован режим генерации пучков убегающих электронов, состоящих из двух импульсов соизмеримой амплитуды. Временной интервал между импульсами составлял десяткисотни ps. Двойные импульсы СЛЭП с длительностью на полувысоте $100-170$ ps регистрировались за $\mathrm{AlMg}$ фольгой толщиной $50 \mu \mathrm{m}$. При увеличении давления оба импульса тока пучка сливались в один.
Кроме цитируемых выше работ, посвященных генерации двух импульсов тока пучка, отметим публикации другой научной группы [30-32]. В этих работах двойные импульсы тока убегающих электронов в воздухе атмосферного давления были получены при экранировке штыревого катода с малым радиусом кривизны дополнительным электродом в виде тора, который имел большой радиус кривизны. Амплитуда падающей волны импульса напряжения составляла $250 \mathrm{kV}$, а фронт импульса $\approx 400$ ps. Задержка между максимумами импульсов в этом режиме составляла $\sim 150$ ps. Энергия электронов во втором импульсе была меньше, чем в первом. Основное отличие результатов работ [30-32] заключалось в том, что при генерации второго импульса, который авторы назвали лавинным пучком, напряжение на промежутке за счет экранировки штыревого катода во время генерации этого импульса и импульса тока пучка существенно не уменьшалось.

Таким образом, результаты известных работ [25-27,30-32] иллюстрируют многообразие условий, при которых в газовых диодах наблюдается генерация двух импульсов тока пучка. Однако механизм генерации двух импульсов тока пучка однозначно не был установлен и исследования пробоя оптическими методами в этих условиях не проводились.

Цель настоящей работы - изучить условия генерации двух импульсов тока пучка во время одного импульса напряжения амплитудой в десятки киловольт с различной длительностью фронта.

Исследования генерации двух импульсов тока пучка были проведены в воздухе, азоте и гелии при регистрации оптических характеристик излучения в газовом диоде с помощью четырехканальной ICCD-камеры и скоростной стрик-камеры. Причем одновременно с оптическим излучением плазмы в промежутке регистрировались импульсы напряжения, тока пучка, тока разряда и динамический ток смещения (ДТС) [33], на английском языке dynamic displacement current (DDC), с пикосекундным временным разрешением.

\section{1. Экспериментальная установка и методики}

Эксперименты проводились на универсальной установке при межэлектродном зазоре $d=8.5$ и $5 \mathrm{~mm}$, которая вместе с регистрирующими датчиками и измерительной аппаратурой показана на рис. 1 .

Напряжение на газовый диод подавалось поочередно от трех высоковольтных генераторов 1 отрицательной полярности через коаксиальную линию с волновым сопротивлением $75 \Omega$ 6. Для этого использовались высоковольтные кабели также с волновым сопротивлением $75 \Omega$ 3. Генераторы работали в моноимпульсном режиме. Напряжение на промежутке измерялось с помощью емкостного делителя напряжения 5. 

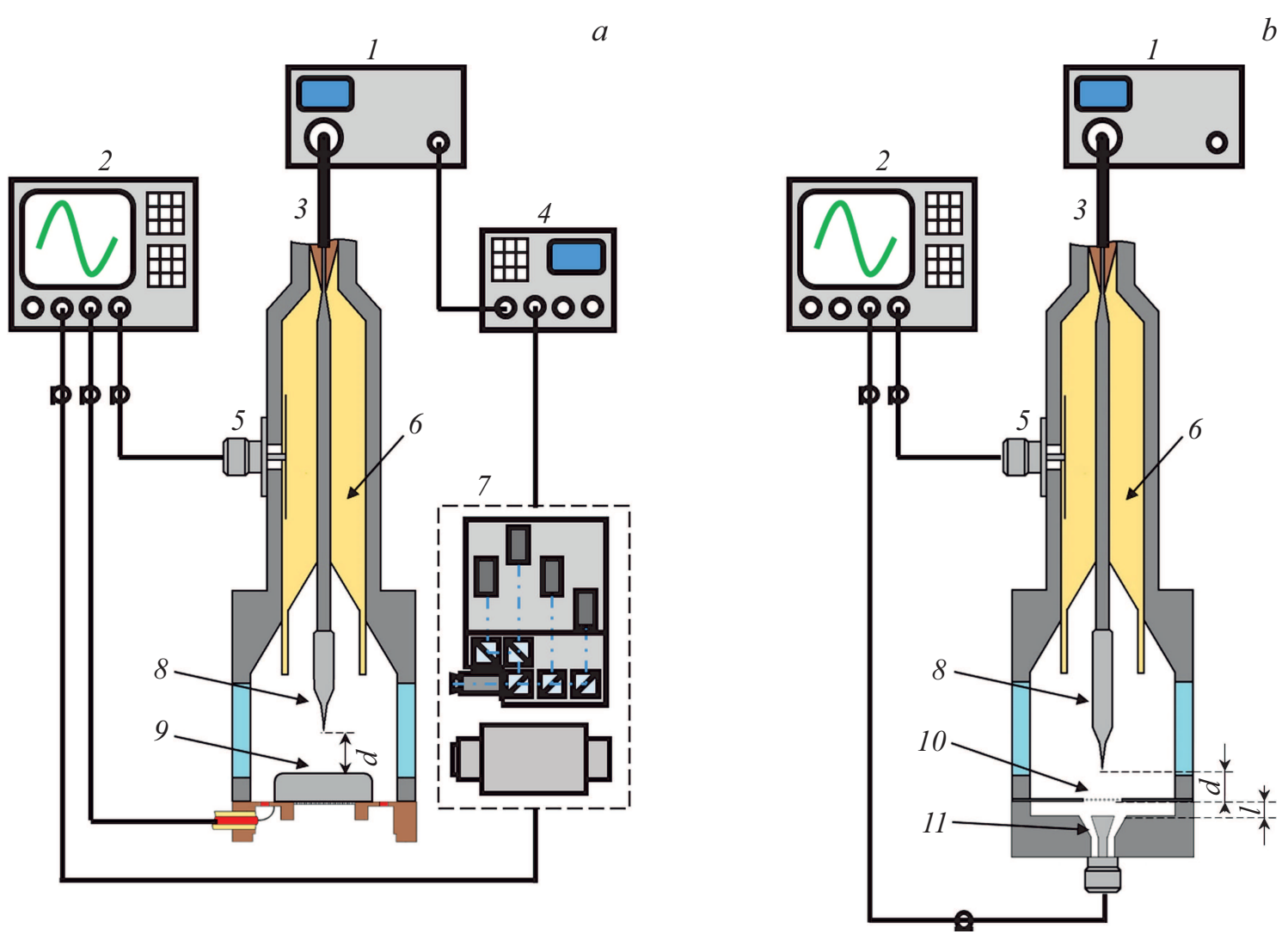

Рис. 1. $a-$ установка для исследования формирования разряда методами высокоскоростной съемки. $b-$ установка для измерения тока убегающих электронов и тока смещения. 1 - высоковольтный генератор, 2 - осциллограф, 3 - высоковольтный кабель, 4 - запускающий генератор, 5 - емкостной делитель напряжения, 6 - коаксиальная линия, 7 - ICCD и стрик-камеры, 8 катод с малым радиусом кривизны, $9-$ плоский анод с шунтом, $10-$ анод с отверстием, закрытым сеткой, $11-$ коллектор.

Динамика свечения плазмы разряда проводилась методами высокоскоростной съемки, рис. 1, $a$. Развитие свечения плазмы разряда вдоль оси разрядного промежутка с разрешением во времени десятки пикосекунд регистрировалось с помощью стрик-камеры Hamamatsu C10910-05 7. С помощью четырехканальной ICCDкамеры HSFC-PRO 7 осуществлялась покадровая съемка разряда с разрешением во времени. Данная камера позволяет получать четыре последовательных изображения за один импульс с минимальной длительность кадра $3 \mathrm{~ns}$ и регулировать задержку между отдельными кадрами c шагом $1 \mathrm{~ns}$. При этом одновременно проводилось измерение напряжения и тока разряда. Изображения с ICCD-камеры были синхронизованы с осциллограммами благодаря регистрации сигнала запуска первого канала камеры. Стрик- и ICCD-камеры запускались от генератора 4. В случае ICCD-камеры запуск регулировался подбором длины линии задержки, а в случае стриккамеры - с помощью задержки импульса в генераторе. Сигналы с датчиков, а также сигнал запуска ICCDкамеры регистрировались осциллографом 2. Данные с камер поступали на компьютер.

Для измерения тока убегающих электронов и динамического тока смещения, вызванного перераспределением напряженности электрического поля в промежутке при формировании и распространении стримера, применялись коллекторы 11 с диаметром приемной части 20, или 7 или $3 \mathrm{~mm}$ (рис. 1, b). Все генераторы формировали импульсы напряжения с регулируемой амплитудой. Генератор ГИН-100-1 [34] (№ 1) имел при максимальной амплитуде напряжения в падающей волне $\approx 35 \mathrm{kV}$ длительность импульса на полувысоте $\tau_{0.5} \approx 4 \mathrm{~ns}$ и длительность фронта $\tau_{0.1-0.9} \approx 0.7 \mathrm{~ns}$. Генератор NPG18 [35] (№ 2) формировал импульс напряжения амплитудой от 13 до $18 \mathrm{kV}$ при $\tau_{0.5} \approx 7$ ns и $\tau_{0.1-0.9} \approx 4$ ns. Генератор GIN-35NP (№ 3), изготовленный в лаборатории, имел максимальную амплитуду напряжения $\approx 35 \mathrm{kV}$, длительность импульса на полувысоте $\tau_{0.5} \approx 270 \mathrm{~ns}$ и длительность фронта $\tau_{0.1-0.9} \approx 120 \mathrm{~ns}$. Высоковольтный электрод 8 длиной $5 \mathrm{~mm}$ был изготовлен из куска швейной иглы, имел диаметр основания $1 \mathrm{~mm}$ и радиус закругления кончика $75 \mu \mathrm{m}$. Игла крепилась к конусу, который плавно переходил в цилиндр диаметром $6 \mathrm{~mm}$ (рис. 1). В ряде экспериментов использовался трубчатый электрод диаметром $2.5 \mathrm{~mm}$, изготовленный из танталовой фольги толщиной $20 \mu \mathrm{m}$. Заземленный электрод 9 был плоским. На установке, показанной на рис. $1, b$, заземленный электрод 10 был изготовлен из медной 
фольги толщиной $0.5 \mathrm{~mm}$, в центре которой имелось отверстие диаметром $10 \mathrm{~mm}$. Отверстие в электроде закрывалось сеткой с размером ячейки $1 \times 1 \mathrm{~mm}$ и пропусканием $67 \%$. В результате электрическое поле частично проникало в пространство между сеткой и коллектором. Величина напряженности электрического поля в этом пространстве определяется величиной напряженности поля вблизи поверхности сетки $E_{c}(t)$. Изменение во времени $E_{c}(t)$ регистрировалось коллектором диаметром $7 \mathrm{~mm}$, как ток смещения. Ток разряда измерялся токовым шунтом, собранным из чип-резисторов. Для оценки энергии убегающих электронов за сеткой помещалась алюминиевая фольга толщиной $10 \mu \mathrm{m}$, либо пленка из кимфоли толщиной $2 \mu \mathrm{m}$, покрытая слоем алюминия толщиной $0.2 \mu \mathrm{m}$.

Сигналы с датчиков 5 и 11, а также токового шунта, регистрировались цифровым осциллографом KeySight MSOS804A (8.4 GHz, 20 GS/s). При измерении тока пучка убегающих электронов применялся коллектор диаметром $3 \mathrm{~mm}$ и использовался осциллограф LeCroy WaveMaster $830 \mathrm{Zi}-\mathrm{A} \quad(16 \mathrm{GHz}, 40 \mathrm{GS} / \mathrm{s})$ с длительностью переходной характеристики до $\approx 26$ ps [36]. Кроме того, перед приемной частью коллектора диаметром $3 \mathrm{~mm}$ устанавливалась дополнительная сетка прозрачностью $30 \%$. В результате напряженность электрического поля ослаблялась в $10^{4}$ раз. Это позволило отсечь ДТС и измерять только ток электронов, долетевших до коллектора.

Разрядная камера была оснащена кварцевыми окнами для вывода излучения.

\section{2. Результаты экспериментов}

Изменение длительности фронта импульса напряжения $\left(\tau_{f}\right)$ выявило следующие закономерности пробоя. Как и следовало ожидать, увеличение $\tau_{f}$ приводило к уменьшению пробойного напряжения, которое при давлении воздуха $25 \mathrm{kPa} \quad(101 \mathrm{kPa}$ при температуре $0^{\circ} \mathrm{C} \approx 1 \mathrm{~atm} \approx 760$ Torr) составило $\sim 50 \mathrm{kV}$ для генератора № 1, $\sim 36 \mathrm{kV}$ для генератора № 2 и $\sim 24 \mathrm{kV}$ для генератора № 3. Со всеми тремя генераторами наблюдалась нестабильность в величине напряжения пробоя от импульса к импульсу, которая влияла на амплитуду тока пучка. Причем с генератором № 2 примерно одинаковое уменьшение амплитуды СЛЭП и ВИТП наблюдалось как при его генерации на фронте, так и на спаде импульса напряжения. При максимальном напряжении на промежутке параметры СЛЭП и ВИТП имели наименьший разброс.

Исследования пробоя в воздухе, азоте и гелии с катодом в форме иглы и $d=8.5 \mathrm{~mm}$ на установке с генераторами № 1-№ 3 позволили реализовать режимы генерации одного СЛЭП, СЛЭП вместе с ВИТП и режим, при котором за СЛЭП продолжительность тока пучка в квазистационарной стадии составляла сотни пикосекунд. Изменение режима генерации достигалось за счет уменьшения давления в газовом диоде. При низких давлениях наблюдался переход к режиму вакуумного диода. При замене катода-иглы на катод в виде трубки генерация ВИТП обычно не наблюдалась. Вероятно, это было обусловлено различием в материале, состоянием кромки и меньшим усилением электрического поля. При уменьшении $d$ с 8.5 до $5 \mathrm{~mm}$ и использовании обоих катодов описанные тенденции сохранялись, но давления, при которых происходила смена режима генерации тока пучка, увеличились.

Было установлено, что изменение $\tau_{f}$, а также сорта газа влияет на давление, при котором фиксируются двойные импульсы тока пучка. Вариация зазора и используемого катода изменяла соотношение амплитуд СЛЭП и ВИТП, а также длительность ВИТП и задержку между первым и вторым импульсами тока пучка. Так, сокращение длительности фронта импульса напряжения, приводящее в данных условиях к увеличению пробойного напряжения, приводило к увеличению давления, при котором появлялись вторые импульсы, и уменьшало задержку между импульсами.

Проиллюстрируем подробно часть из описанных тенденций. Осциллограммы СЛЭП, СЛЭП вместе с ВИТП и ДТС, полученные с генератором № 2, вместе с импульсами напряжения при разных давлениях воздуха приведены на рис. 2.

При измерении ДТС, как уже было отмечено выше, применялся коллектор диаметром $7 \mathrm{~mm}$, а при измерении тока пучка электронов - коллектор диаметром $3 \mathrm{~mm}$. При этом перед приемной частью коллектора устанавливалась дополнительная сетка прозрачностью $30 \%$, для того чтобы отсечь ДТС (за сеткой напряженность электрического поля была в $\sim 10^{4}$ раз меньше).

Имеющийся осциллограф и коллекторы позволили с высоким временным разрешением измерить ток пучка электронов и ДТС в различных режимах. Первое, что необходимо отметить - это воспроизводимость характеристик разряда. Это было важно для совмещения тока пучка электронов и ДТС, которые измерялись в разных импульсах. В каждом эксперименте записывалось 30 осциллограмм. Затем выбирались осциллограммы с одинаковым запаздыванием пробоя. Видно (рис. 2), что если процессы ионизации начинаются при одном значении напряжения, то динамика пробоя очень хорошо воспроизводится. Воспроизводятся даже мельчайшие колебания на осциллограмме напряжения (рис. 2, $a-c, e)$. Второе, что необходимо пояснить, осциллограмма ДТС весьма точно показывает, когда происходит старт волны ионизации и когда она прибывает на противоположный электрод. Подробно об этом написано в работе [33]. В настоящей работе отметим только, что начало ДТС соответствует старту первой волны ионизации, а момент, когда происходит первое изменение полярности сигнала, соответствует прибытию первой волны ионизации на противоположный сетчатый 

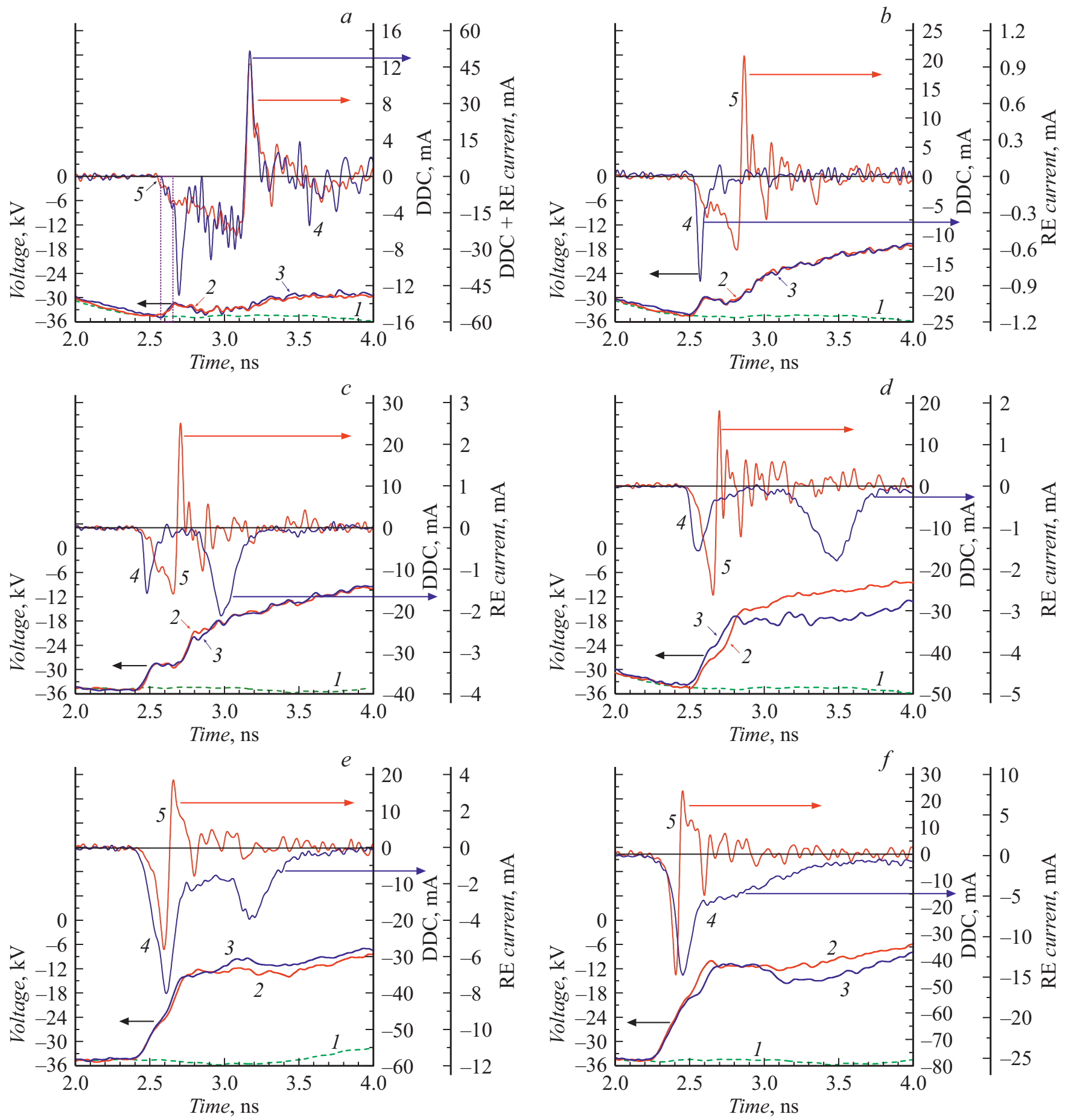

Рис. 2. Осциллограммы напряжения в режиме холостого хода $(1)$ и при разряде $(2,3)$, а также осциллограммы тока убегающих электронов (4), измеренного коллектором, и динамического тока смещения (5), также измеренного коллектором. Осциллограмма напряжения (2) получена в эксперименте по измерению динамического тока смещения, а осциллограмма напряжения (3) - в эксперименте по измерению тока убегающих электронов. Воздух при давлении: $100(a), 50(b), 25(c), 12(d), 6(e), 3(f) \mathrm{kPa}$. Кривая (4) при давлении воздуха $100 \mathrm{kPa}(a)$ получена при одновременном измерении тока убегающих электронов и динамического тока смещения. Генератор № 2, промежуток игла-плоскость, $d=8.5 \mathrm{~mm}$.

электрод. Этот момент соответствует резкому уменьшению напряженности электрического поля (если рассматривать интеграл от сигнала ДТС) вблизи сетчатого электрода вследствие ионизации газа.
В-третьих, на рис. 2, а показана осциллограмма, полученная в условиях, когда токи СЛЭП и ДТС измеряются коллектором одновременно. Эта осциллограмма позволяет определить с высокой точностью момент ге- 

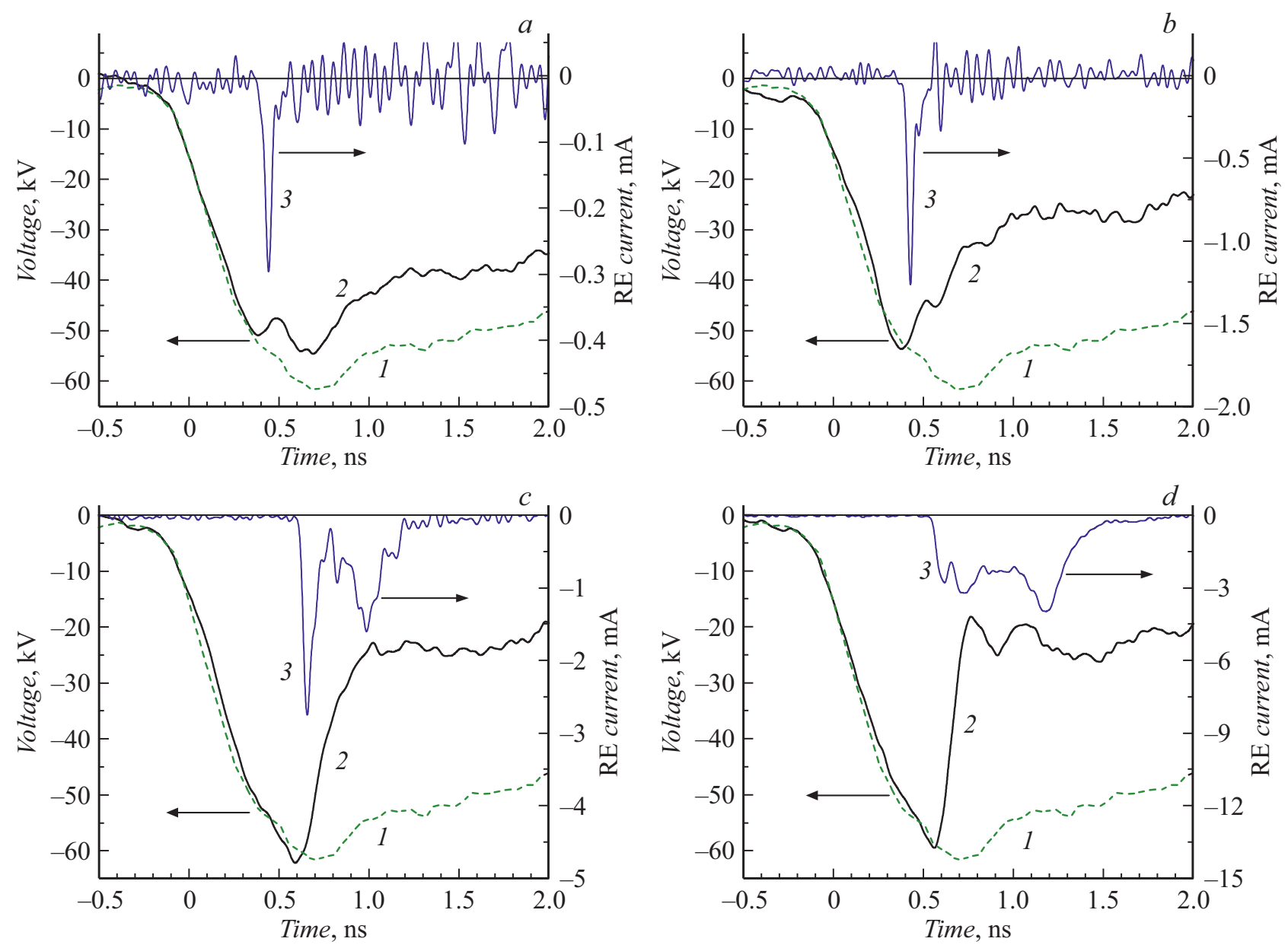

Рис. 3. Осциллограммы напряжения в режиме холостого хода (1) и при разряде (2), а также осциллограммы тока убегающих электронов. Азот при давлении $100(a), 50(b), 25(c)$ и $12(d) \mathrm{kPa}$. Генератор № 1, промежуток игла-плоскость, $d=8.5$ mm.

нерации убегающих электронов относительно динамики волны ионизации. Для этого необходимо также учесть время пролета пучка электронов до коллектора и время распространения электромагнитной волны, вызывающей сигнал ДТС. Оценки показывают, что импульс тока СЛЭП на рис. 2, $a$ должен быть сдвинут влево как минимум на 74 ps. Таким образом, момент генерации СЛЭП соответствует старту волны ионизации (на рис. 2 , a момент генерации СЛЭП показан вертикальными пунктирными линиями). Осциллограммы тока пучка, представленные на рис. $2, b-f$, сдвинуты влево с учетом времени пролета пучка электронов до коллектора. При этом важно отметить, что форма и амплитуда тока пучка, зарегистрированного коллектором, может несколько отличаться от того, что было в момент генерации вблизи катода. Это связано с тем, что электроны в пучке имеют различную энергию, а также они испытывают упругие и неупругие столкновения.

В-четвертых, из рис. 2 видно, что старт волны ионизации и ее распространение в направлении противоположного электрода сопровождаются спадом напряжения на промежутке. Чем ниже давление газа, тем выше скорость волны ионизации и тем больше сигнал ДТС и сильнее спад напряжения на промежутке.

Генерация ВИТП в воздухе с генератором № 2 и промежутком игла-плоскость с $d=8.5 \mathrm{~mm}$ наблюдалась при давлении $\sim 25 \mathrm{kPa}$ и ниже. Напряжение на промежутке во время генерации второго импульса тока пучка было вдвое меньше начального и существенно не изменялось во время всей длительности ВИТП. Средняя приведенная напряженность электрического поля во время второго импульса (рис. 2,c) не превышала $130 \mathrm{kV} / \mathrm{cm} \cdot$ Torr. Уменьшение давления в данных условиях могло приводить к увеличению задержки между СЛЭП и ВИТП (рис. $2, d, e)$. При заполнении газового диода азотом наблюдались подобные зависимости. В гелии одновременная генерация СЛЭП и ВИТП сохранялась и при более высоком давлении $(\approx 100 \mathrm{kPa})$. Использование трубчатого катода уменьшило давления, при которых наблюдалась одновременная генерация СЛЭП и ВИТП.

Сокращение длительности фронта импульса напряжения увеличило напряжение пробоя (рис. 3) и уменьшило временной интервал между максимумами СЛЭП и 

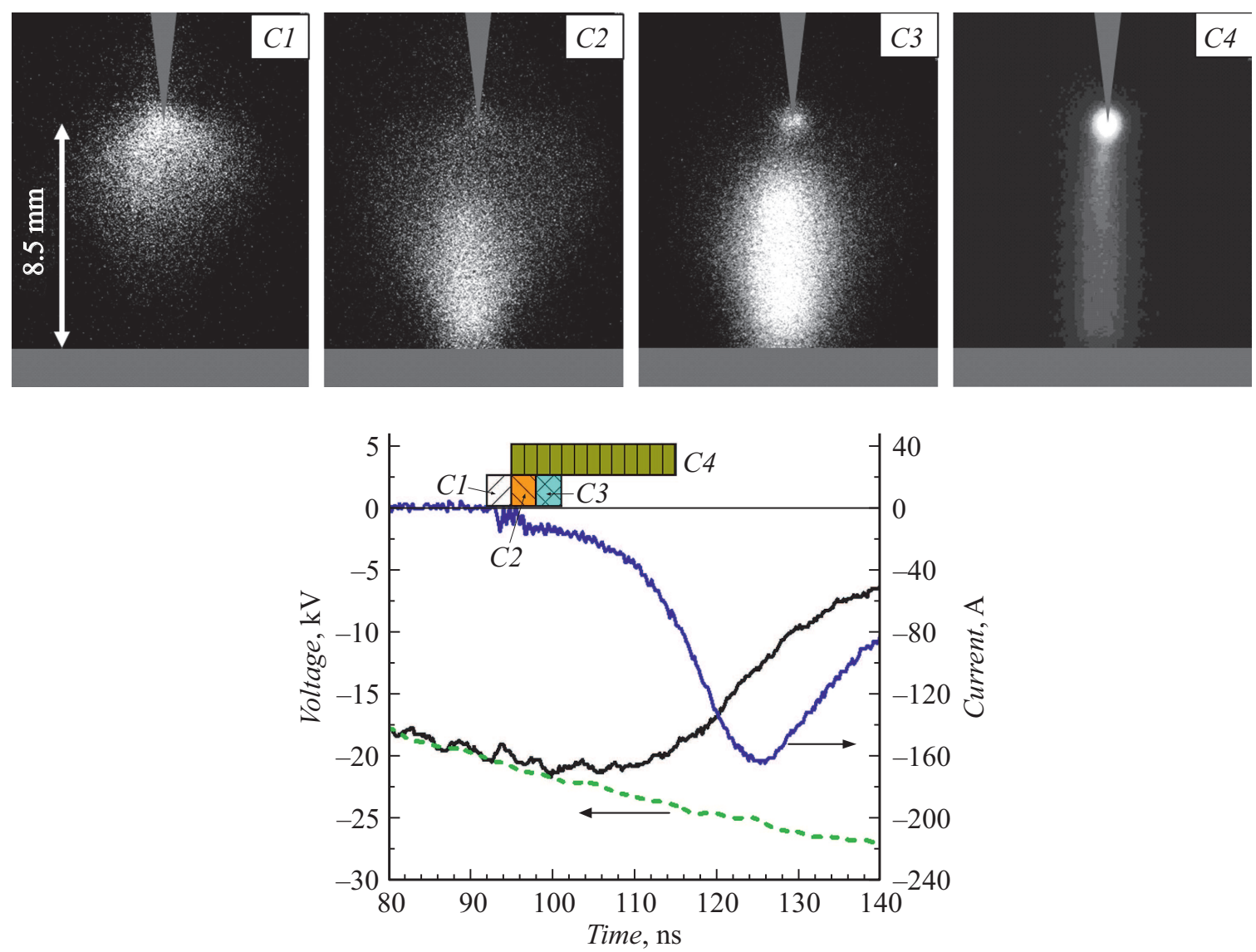

Рис. 4. Изображения, демонстрирующие формирование разряда в азоте при давлении $100 \mathrm{kPa}$, полученные с помощью четырехканальной ICCD-камеры за один импульс, а также соответствующие осциллограммы напряжения и тока. Длина прямоугольников соответствует длительности экспозиции каждого канала ICCD-камеры $(C 1-C 4)$. Генератор № 3. Пунктиром показано напряжение холостого хода.

ВИТП до $\approx 320$ рs при давлении $25 \mathrm{kPa}$ (рис. $3, c$ ). Эта задержка стала меньше, чем при том же давлении с генератором № 2 (рис. 2,c). При давлении $12.5 \mathrm{kPa}$ за первым импульсом появилась квазистационарная стадия тока пучка (рис. $3, d$ ). Напряжение на промежутке после быстрого спада существенно не изменялось. С генератором № 2 подобный режим наблюдался при давлении азота $3 \mathrm{kPa}$ (рис. 2,f).

Увеличение длительности фронта импульса напряжения (генератор № 3) уменьшило давление воздуха, при котором регистрировались одновременно СЛЭП и ВИТП с амплитудой второго импульса большей, чем первого, см. также [27]. Сравнение условий получения СЛЭП и ВИТП в воздухе и азоте с генератором № 3 подтвердило, что они существенно не отличаются.

Исследование динамики свечения плазмы в промежутке при генерации СЛЭП и ВИТП проводилось в азоте и воздухе с помощью ICCD и стрик-камер. Наибольшая яркость интегрального излучения плазмы в спектральном диапазоне, регистрируемом ICCD- и стриккамерами, наблюдалась в азоте. На рис. 4 приведены фотографии свечения промежутка, полученные на ICCDкамере, и осциллограммы тока разряда и напряжения на промежутке с указанием времени съемки отдельных кадров.

Для наглядной иллюстрации различных стадий пробоя промежутка приведено свечение азота при давлении $100 \mathrm{kPa}$ с импульсом напряжения от генератора № 3. При увеличении давления и уменьшении напряжения стадии формирования стримера растягиваются во времени, а скорость движения его фронта уменьшается. Также уменьшается скорость распространения последующих волн ионизации. В этих условиях их динамика развития разряда хорошо различима при использовании имеющейся ICCD-камеры.

На первом кадре (рис. 4, C1), виден стример шаровой формы [9], фронт которого достиг половины разрядного промежутка. На осциллограмме тока разряда, которая приведена на графике под изображениями разряда, в это время регистрируется первый пик ДТС. На втором кадре (рис. 4,C2) показано, что стример пересек разрядный промежуток, и вторая волна ионизации удалилась от анода. Увеличение тока разряда на этом кадре соответствует времени прихода фронта стримера на анод. Третий кадр (рис. 4,C3) показывает, что вторая волна ионизации приблизилась к катоду и яркость ее свечения увеличилась. Во время третьего кадра на катоде в этих 


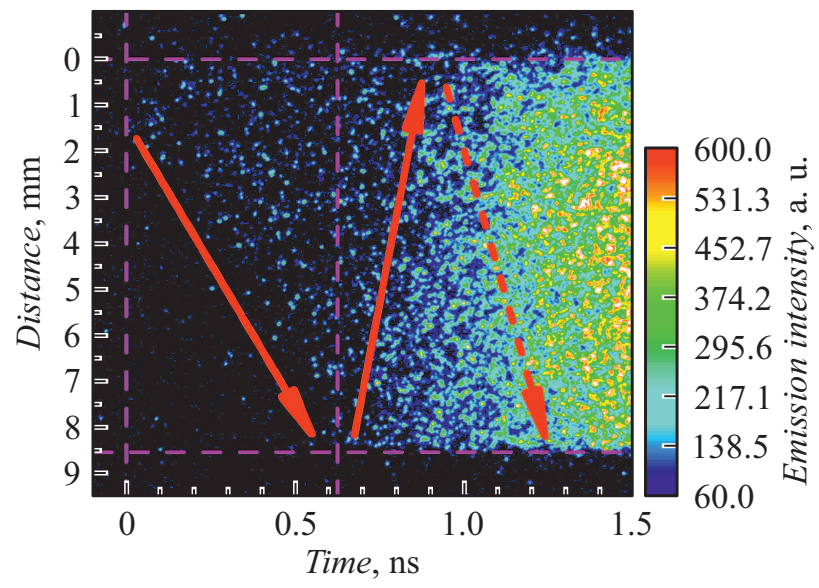

Рис. 5. Изображение, демонстрирующее динамику свечения плазмы при формировании разряда в промежутке игла(сверху)-плоскость(внизу), заполненном воздухом при давлении $50 \mathrm{kPa}$. Генератор № 2. Стрелками показано направление движения волн ионизации.

условиях появилась область яркого свечения, которое возникло в результате взрывной эмиссии электронов. На четвертом кадре (интенсивность свечения плазмы ослаблена относительно свечения катодного пятна) показано интегральное свечение диффузного разряда за $20 \mathrm{~ns}$ (рис. 4, C4). Ширина сформировавшегося диффузного разряда меньше максимального диаметра стримера, а яркость его излучения больше, чем у стримера. При длительности кадра $20 \mathrm{~ns}$ на фотографии доминирует свечение яркого катодного пятна, появившегося на третьем кадре.

С уменышением давления время развития первой и второй (обратной) волн ионизации сокращалось. Полагаем, что с уменьшением давления в диоде динамика пробоя качественно не изменяется.

Эксперименты с ICCD также показали, что при давлении $25 \mathrm{kPa}$ с генератором № 2 в отсутствии яркого пятна на катоде регистрировались СЛЭП и ВИТП. Также СЛЭП и ВИТП регистрировались при появлении яркого пятна у катода с задержкой более 2 ns после старта стримера с катода, однако при появлении катодного пятна до перекрытия промежутка стримером регистрировался только СЛЭП. Подобная динамика свечения разряда наблюдается и с генератором № 3. При увеличении напряжения (генератор № 1) или(и) уменьшении давления газа скорость движения фронта волны ионизации возрастала и временного разрешения данной ICCD-камеры не хватало для сопоставления динамики свечения плазмы в промежутке с осциллограммами ДТС и пучка электронов с коллектора.

Свечение промежутка, полученное с помощью стриккамеры, подтверждает данные о формировании нескольких волн ионизации, которые были зарегистрированы ICCD-камерой. На рис. 5 приведена временна́я развертка свечения разряда в воздухе для давления $50 \mathrm{kPa}$.
Временное разрешение стрик-камеры составляет единицы-десятки пикосекунд, но яркость свечения плазмы в первую наносекунду пробоя очень мала. На приведенной фотографии стрелками показано движение волн ионизации в промежутке. Видна корреляция между свечением промежутка и ДТС-сигналом, регистрируемым коллектором (рис. 2, b). Наибольшую среднюю скорость в этих условиях имела вторая волна ионизации. Предварительная ионизации промежутка для нее была осуществлена СЛЭП и первой волной. Кроме того, на промежутке сохранилось сравнительно высокое напряжение. Скорость третьей волны, из-за значительного уменьшения напряжения на промежутке относительно начального, была меньше, чем у второй.

При анализе полученных данных следует иметь в виду следующие особенности измерений. Во-первых, время высвечивания второй положительной системы молекул азота, излучение которой дает основной вклад в сигналы c ICCD- и стрик-камер, зависит от давления, что затрудняет регистрацию скорости движения фронта волны ионизации, особенно, с уменьшением давления. Как известно, при $p=100 \mathrm{kPa}$ время высвечивания молекулы азота в УФ и видимой областях спектра на переходах второй положительной системы азота составляет $\sim 1 \mathrm{~ns}$, а при давлении $30 \mathrm{kPa}-\sim 4$ ns. Во-вторых, при малых концентрациях плазмы в промежутке ДТС не регистрируется. Для регистрации динамического емкостного тока концентрация заряженных частиц плазмы в промежутке должна превышать пороговую, которую легче получить при повышенных давлениях газа в области максимального электрического поля у острия. В-третьих, надо учитывать время пролета убегающих электронов до анода, а также в области между анодом и коллектором.

\section{3. Обсуждение результатов}

Из полученных с помощью ICCD- и стрик-камер данных следует, что пробой промежутка происходит за счет волн ионизации, скорость которых увеличивается с уменьшением давления и ростом напряжения на промежутке. Подобные тенденции наблюдались при пробое в длинных трубках [28], а также при исследованиях стримерных разрядов [37-39].

Генерация СЛЭП начинается в начальной стадии пробоя промежутка, примерно соответствуя началу регистрации динамического емкостного тока, см. также работы $[33,40]$. Из этого следует, что первые эмитированные электроны инициируют волну ионизации и часть этих, а также часть вторичных электронов, становятся убегающими. Для перехода электронов в режим убегания необходимо достигнуть достаточно высокого значения параметра $E / N$, где $N-$ концентрация частиц газа. При комнатной температуре газов вместо $E / N$ часто используют параметр $E / p$. Следует отметить, что критическое поле $E_{\mathrm{cr} 1}$, достаточное для появления небольшого числа убегающих электронов, и $E_{\mathrm{cr} 2}$, необходимое для убегания основного числа электронов из 
разрядной плазмы с учетом их размножения, существенно различаются. В первом случае $E_{\text {cr1 }} / p$ составляет для азота около $590 \mathrm{~V} / \mathrm{cm} \cdot$ Torr (см. [41]), а во втором $E_{\mathrm{cr} 2} / p \approx 4 \mathrm{kV} / \mathrm{cm} \cdot$ Torr (см. [41]). Отметим также работы $[42,43]$, где было предложено использовать нелокальный критерий убегания электронов. Существенное увеличение электрического поля в промежутке, которое следует из нелокального критерия, необходимо для перехода в режим убегания вторичных электронов с низкими энергиями, появившихся в результате лавинного размножения. Это позволяет существенно увеличить амплитуду СЛЭП $[9,12]$.

Для перехода в режим убегания при относительно малых электрических полях очень важно, чтобы электроны быстро набрали начальную энергию до $\sim 1 \mathrm{keV}$ и более. Для этого важно применять катоды с малым радиусом кривизны [3], что приводит к значительному усилению локального электрического поля. Известно, см., например, $[5,6,44,45]$, что с ростом энергии электрона $>0.1 \mathrm{keV}$ в азоте, воздухе и других газах, потери их энергии на ионизацию и возбуждение уменьшаются вплоть до энергий $\sim 1 \mathrm{MeV}$. Поэтому при увеличении энергии электронов их ускорение может продолжаться даже при $E / p<E_{\mathrm{cr} 1} / p$. Соответственно для реализации режима убегания необходимо, чтобы электроны получили начальное ускорение.

С катодом в форме иглы поле у острия с генераторами № 1 и № 2 превышает $1 \cdot 10^{6} \mathrm{~V} / \mathrm{cm}$. Поскольку на катоде имеются микровыступы, а также диэлектрические пленки [46] и наночастицы [47], которые дополнительно усиливают электрическое поле, порог автоэлектронной эмиссии достигается при меньших макрополях по сравнению с автоэлектронной эмиссией в вакууме. Часть эмитированных с катода электронов, а также часть вторичных электронов, полученных в результате ионизации газа, уходят в режим непрерывного ускорения вблизи катода и начинают формировать первый пучок электронов - СЛЭП. Электроны, которые имеют сравнительно малые энергии, дают начало одной или нескольким лавинам, головки которых перекрываются. В результате, за короткое время на расстоянии в сотни $\mu \mathrm{m}$ от катода возникает стример. Создаваемая им и лавинами плазма испускает коротковолновое излучение, что сохраняет эмиссию электронов с катода при уменьшении электрического поля за счет его экранировки плотной плазмой. Основное отличие стримера от лавины - это существенное усиление напряженности электрического поля на его фронте. Измерения, проведенные в работах $[48,49]$, показали, что напряженность электрического поля на головке стримера в воздухе атмосферного давления при напряжениях 20-85 kV составляет $\sim 200 \mathrm{kV} / \mathrm{cm}$ и зависит от положения стримера в промежутке. Благодаря этому, электроны, двигаясь синхронно с фронтом стримера (волны ионизации), могут набирать дополнительную энергию. Данный эффект был теоретически предсказан Аскарьяном [50] и используется для объяснения генерации электронов $\mathrm{c}$,аномальной энергией“ (большей $e U_{m}$, где $e-$ заряд электрона, а $U_{m}-$ максимальное напряжение на промежутке) $[12,23,51,52]$.

В данных условиях электроны СЛЭП генерируются в момент времени, близкий к моменту достижения напряжением на промежутке максимального значения, и опережают фронт стримера при его движении к аноду. Соответственно электроны СЛЭП некоторое время движутся между анодом и фронтом стримера с отрицательным зарядом. Это приводит к дополнительному усилению поля для этих электронов. Соответственно СЛЭП может генерироваться при более высоких давлениях воздуха и других газов по сравнению с ВИТП. Так, с генератором № 1 СЛЭП был получен при давлении воздуха $100 \mathrm{kPa}$ за анодом из $\mathrm{Al}$ фольги толщиной $10 \mu \mathrm{m}$. Для генерации заметного числа электронов с „аномальной“ энергией следует увеличивать радиус катода и использовать субнаносекундный фронт импульса напряжения. Так, при использовании сферического катода с радиусом кривизны $9.5 \mathrm{~mm}$ и генератора СЛЭП150 доля электронов с ,аномальной“ энергией в пучке составила $\sim 10 \%[9,12]$.

Второй импульс тока пучка, как видно из рис. 2 и 3, появляется с задержкой после первого. Генерация ВИТП происходит после касания фронтом стримера анода и при более низких средних $E / p$, чем СЛЭП. В это время катод уже экранирован плотной плазмой, и величина $E / p$ вблизи него уменьшилась. Это подтверждают измерения распределения электрического поля в промежутке при движении стримера для подобных условий в $[48,49]$. Считаем, что генерация ВИТП происходит благодаря протеканию двух процессов. Это сохранение относительно высокого электрического поля в прикатодном слое за счет положительных ионов и усиление электрического поля на фронте второй волны ионизации. Как известно, усиление поля за счет положительных ионов обеспечивает в стационарном режиме получение быстрых электронов в прикатодной области тлеющего разряда и при постоянном напряжении. Из рис. 2 и 3 видно, что напряжение на промежутке во время импульса ВИТП существенно не изменяется, но ВИТП имеет короткую длительность. Однако только этим процессом нельзя объяснить генерацию ВИТП. Также надо учесть усиление электрического поля на фронте второй волны ионизации, которая движется от анода. Соответственно генерации ВИТП обусловлены, во-первых, эмиссией электронов с катода под действием коротковолнового излучения из плазмы, и, возможно, дают вклад ионы и быстрые нейтральные частицы [53]. Во-вторых, последовательным ускорением электронов в прикатодном слое, а затем в поле фронта второй волны ионизации. Как обсуждалось выше, ВИТП отсутствует при появлении у катода плотной плазмы за счет взрывной эмиссии электронов, которая уменьшает электрическое поле в этой области, и ВИТП имеет максимум из-за влияния второй волны ионизации. Измерения электрического поля на фронте стримера (волны ионизации) при напряжении $\approx 25 \mathrm{kV}$ дали для 
воздуха его величину в подобных условиях, не превышающую $\sim 200 \mathrm{kV} / \mathrm{cm}[48,49]$. Из этого следует, что без ускорения электронов из прикатодной области усиления электрического поля на фронте второй волны ионизации не хватит для получения ВИТП. Для генерации ВИТП также необходим предварительный набор энергии электронами в электрическом поле у катода.

Отметим, что положительное и отрицательное влияние взрывной электронной эмиссии на генерацию СЛЭП теоретически обсуждалось в работе [54], но генерация ВИТП в этой работе не рассматривалась. Расчеты для условий работ [25,26], проведенные в [55], показали, что задержка пробоя и связанного с ним второго наносекундного пучка убегающих электронов связана с задержкой эмиссии электронов с катода. Однако фотоэмиссия в этой настоящей работе не рассматривалась. Полученные экспериментальные данные показывают, что генерацию второго импульса тока пучка ограничивает взрывная эмиссия электронов, а генерация первого импульса может продолжаться после достижения анода фронтом первой волны ионизации, рис. $2, d-f$.

С увеличением средних значений $E / p$ до $\sim 300 \mathrm{kV} / \mathrm{cm}$ и более генерация убегающих электронов продолжалась без паузы после генерации СЛЭП. Наблюдался квазистационарный режим генерации пучка электронов с увеличенной длительностью импульса как с генератором № 1 (рис. 3,d), так и с генератором № 2 (рис. $2, f)$. Увеличение $E / p$ в данных условиях достигалось за счет уменьшения давления газа. Этот режим соответствует квазистационарному режиму аномального тлеющего разряда. Электроны ускоряются в катодном слое и достигают коллектора. Дальнейшее уменьшение давления газа приводит к переходу в режим генерации тока пучка, который реализуется в вакуумных диодах.

\section{Заключение}

Проведенные исследования показали, что вторые импульсы тока пучка убегающих электронов наблюдаются при различной длительности фронта импульса напряжения. Их появление при фиксированной амплитуде импульса напряжения определяется давлением и сортом газа, а также формой катода. Для генерации вторых импульсов тока пучка, которые регистрируются после прохождения первой волны ионизации и уменьшения напряжения на промежутке, необходимо дополнительное усиление электрического поля. Это достигается при низких средних $E / p$ в двух областях - за счет накопления положительных ионов у катода и за счет увеличения электрического поля на фронте второй волны ионизации, которая стартует от анода. Вторая волна ионизации имеет высокую проводимость, что следует из интенсивности ее свечения, и обеспечивает частичный вынос потенциала анода в прикатодную область. Фактором, ограничивающим генерацию второго импульса тока пучка, в данных условиях является взрывная эмиссия электронов, которая приводит к уменьшению напряженности электрического поля в прикатодном слое.
Переходный к условиям вакуумного диода режим генерации тока пучка реализуется при сравнительно высоких средних $E / p$ за счет существенного уменьшения давления газа. В этом режиме после первого импульса тока пучка без паузы регистрируется квазистационарный пучок электронов с длительностью $\sim 1 \mathrm{~ns}$, амплитуда которого увеличивается с сокращением фронта импульса напряжения.

\section{Финансирование работы}

Исследование выполнено при финансовой поддержке РФФИ в рамках научного проекта № 20-02-00733.

\section{Конфликт интересов}

Авторы заявляют, что у них нет конфликта интересов.

\section{Список литературы}

[1] C.T.R. Wilson. Proc. Phys. Soc. London, 37, 32D (1924).

[2] H. Dreicer. Phys. Rev., 115, 238 (1959).

[3] S. Frankel, V. Highland, T. Sloan, O. Van Dyck, W. Wales. Nucl. Instrum. Methods, 44, 345 (1966).

[4] Ю.Л. Станкевич, В.Г. Калинин. ДАН СССР, 177 (1), 72 (1967). [Y.L. Stankevich, N.S. Kalinin. Sov. Phys. Dokl., 1, 72 (1967).]

[5] A.V. Gurevich, G.M. Milikh, R. Roussel-Dupre. Physics Letters A, 165 (5-6), 463 (1992). doi.org/10.1016/0375-9601(92)90348-P

[6] T.F. Bell, V.P. Pasko, U.S. Inan. Geophys. Res. Lett., 22 (16), 2127 (1995). doi.org/10.1029/95GL02239

[7] Л.В. Тарасова, Л.Н. Худякова, Т.В. Лойко, В.А. Цукерман. ЖТФ, 44 (3) 564 (1974). [L.V. Tarasova, L.N. Khudyakova, T.V. Loiko, V.A. Tsukerman. Sov. Phys. Tech. Phys., 19, 351 (1974).]

[8] П.А. Бохан, Г.В. Колбычев. Письма в ЖТФ, 6 (7), 418 (1980). [P.A. Bokhan, G.V. Kolbychev, Sov. Tech. Phys. Lett., 6, 418 (1980).

[9] V. Tarasenko. Plasma Sources Sci. Technol., 29 (3), 034001 (2020). doi.org/10.1088/1361-6595/ab5c57

[10] G.A. Mesyats, M.I. Yalandin, N.M. Zubarev, A.G. Sadykova, K.A. Sharypov, V.G. Shpak, S.A. Shunailov, M.R. Ulmaskulov, O.V. Zubareva, A.V. Kozyrev, N.S. Semeniuk. Appl. Phys. Lett., 116 (6), 063501 (2020). doi.org/10.1063/1.5143486

[11] N.A. Ashurbekov, K.O. Iminov, O.A. Popov, G.S. Shakhsinov. Plasma Sci. Technol., 19 (3), 035401 (2017). DOI: $10.1088 / 2058-6272 / 19 / 3 / 035401$

[12] V.F. Tarasenko, E.Kh. Baksht, A.G. Burachenko, I.D. Kostyrya, M.I. Lomaev, D.V. Rybka. Plasma Devises and Operation, 16 (4), 267 (2008). DOI: 10.1080/10519990802478847

[13] Ю.С. Акишев, А.А. Балакирев, В.Б. Каральник, М.А. Медведев, А.В. Петряков, Н.И. Трушкин, А.Г. Шафиков. Физика, 60 (8), 70 (2017). [Y.S. Akishev, A.A. Balakirev, V.B. Karal'nik, M.A. Medvedev, A.V. Petryakov, N.I. Trushkin, A.G. Shafikov. Rus. Phys. J., 60 (8), 1341 (2017).] DOI: $10.1007 / \mathrm{s} 11182-017-1219-\mathrm{Z}$

[14] T. Shao, R. Wang, C. Zhang, P. Yan. High Voltage, 3 (1), 14 (2018). DOI: 10.1049/hve.2016.0014

[15] W. Jiang, K. Yatsui, V.M. Orlovskii, V.F. Tarasenko. Proceedings of Int. Conf. BEAMS-2004. Saint-Peterburg. 174 (2005). 
[16] А.Н. Ткачев, А.А. Феденев, С.И. Яковленко. ЖТФ, 77 (6), 22 (2007). [A.N. Tkachev, A.A. Fedenev, S.I. Yakovlenko. Tech. Phys., 52 (6), 699 (2007).] doi.org/10.1134/S1063784207060047

[17] S.Y. Belomyttsev, A.A. Grishkov, V.A. Shklyaev, V.V. Ryzhov. J. Appl. Phys., 123, 043309 (2018). doi.org/10.1063/1.5008820

[18] S. Yatom, D. Levko, J.Z. Gleizer, V. Vekselman, Y.E. Krasik. J. Appl. Phys. Lett., 100 (2), 024101 (2012). DOI: $10.1063 / 1.3675462$

[19] V.Y. Kozhevnikov, A.V. Kozyrev, N.S. Semeniuk, A.O. Kokovin. IEEE Transactions on Plasma Sci., 46 (10), 3468 (2018). DOI: 10.1109/TPS.2018.2866777

[20] Н.М. Зубарев, С.Н. Иванов. Физика плазмы, 44 (4), 397 (2018). [N.M. Zubarev, S.N. Ivanov. Plasma Phys. Reports, 44, 445 (2018).] DOI: 10.1134/S1063780X18040104

[21] N.Y. Babaeva, G.V. Naidis, D.V. Tereshonok, E.E. Son. J. Physics D: Appl. Phys., 51, 434002 (2018). doi.org/10.1088/1361-6463/aada74

[22] Л.П. Бабич, Т.В. Лойко, Б.Н. Шамраев. Известия вузов. Радиотехника, 22 (1), 100 (1979). [L.P. Babich, T.N. Loiko, B.N. Shamraev. Radio Phys. Quant. Electron. 22, 68 (1979).]

[23] Л.П. Бабич. Физика плазмы, 8 (4), 718 (1982). [L.P. Babich. Sov. J. Plasma Phys., 8, 404 (1982).]

[24] К.И. Алмазова, А.Н. Белоногов, В.В. Боровков, Е.В. Горелов, И.В. Морозов, А.А. Тренькин, С.Ю. Харитонов. ЖТФ, $88(6), 827$ (2018). [K.I. Almazova, A.N. Belonogov, V.V. Borovkov, E.V. Gorelov, I.V. Morozov, A.A. Tren'kin, S.Y. Kharitonov. Tech. Phys., 63(6), 801 (2018).] DOI: 10.1134/S1063784218060026

[25] Е.Х. Бакшт, В.Ф. Тарасенко, М.И. Ломаев, Д.В. Рыбка. Письма в ЖТФ, 33 (9), 29 (2007). [Е.K. Baksht, V.F. Tarasenko, M.I. Lomaev, D.V. Rybka. Tech. Phys. Lett., 33 (5), 373 (2007).] doi.org/10.1134/S1063785007050045

[26] E.Kh. Baksht, V.F. Tarasenko, M.I. Lomaev, D.V. Rybka, A.N. Tkachev, S.I. Yakovlenko. Laser Phys., 17 (9), 1124 (2007). doi.org/10.1134/S1054660X07090034

[27] V. Tarasenko, D. Beloplotov, M. Lomaev, D. Sorokin. Plasma Sci. Technol., 21 (4), 044007 (2019). doi.org/10.1088/2058-6272/ab079b

[28] Л.М. Василяк, С.В. Костюченко, Н.Н. Кудрявцев, И.В. Филюгин. УФН, $164(3), 263$ (1994). [L.M. Vasilyak, S.V. Kostyuchenko, N.N. Kudryavtsev, I.V. Filyugin. PhysicsUspekhi, 37 (3), 247 (1994).] doi.org/10.1070/PU1994v037n03ABEH000011

[29] В.Ф. Тарасенко, Д.А. Сорокин, М.И. Ломаев. ЖТФ, 86 (10), 109 (2016). [V.F. Tarasenko, D.A. Sorokin, M.I. Lomaev. Tech. Phys., 61 (10), 1551 (2016).] doi.org/10.1134/S106378421610025X

[30] A.V. Gurevich, G.A. Mesyats, K.P. Zybin, A.G. Reutova, V.G. Shpak, S.A. Shunailov, M.I. Yalandin. Phys. Lett. A, 375, 2845 (2011). doi.org/10.1016/j.physleta.2011.06.004

[31] A.V. Gurevich, G.A. Mesyats, K.P. Zybin, M.I. Yalandin, A.G. Reutova, V.G. Shpak, S.A. Shunailov. Phys. Rev. Lett., $109(8), 085002$ (2012). doi.org/10.1103/PhysRevLett.109.085002

[32] G.A. Mesyats, A.G. Sadykova, S.A. Shunailov, V.G. Shpak, M.I. Yalandin. IEEE Trans. Plasma Sci., 10 (41), 2863 (2013). DOI: 10.1109/TPS.2013.2258041

[33] Д.В. Белоплотов, М.И. Ломаев, В.Ф. Тарасенко, Д.А. Сорокин. Письма в ЖЭТФ, 107 (10), 636 (2018). [D.V. Beloplotov, M.I. Lomaev, D.A. Sorokin, V.F. Tarasenko. JETP Lett., 107, 606 (2018).] doi.org/10.1134/S0021364018100065
[34] V.M. Efanov, M.V. Efanov, A.V. Komashko, A.V. Kirilenko, P.M. Yarin, S.V. Zazoulin. Ultra-Wideband, Short Pulse Electromagnetics 9 (Springer, Berlin, 2010), p. 5.

[35] A.G. Lyublinsky, S.V. Korotkov, Y.V. Aristov, D.A. Korotkov. IEEE Trans. Plasma Sci., 41, 2625 (2013). DOI: $10.1109 /$ TPS.2013.2264328

[36] V.F. Tarasenko, D.V. Rybka, A.G. Burachenko, M.I. Lomaev, E.V. Balzovsky. Rev. Sci. Instrum., 83, 086106 (2012). doi.org/10.1063/1.4746378

[37] A.Yu. Starikovskii, A.A. Nikipelov, M.M. Nudnova, D.V. Roupassov. Plasma Sources Sci. Technol., 18 (3), 034015 (2009). DOI: 10.1088/0963-0252/18/3/034015A

[38] D. Wang, T. Namihira. Plasma Sources Sci. Technol., 29 (2), 023001 (2020). doi.org/10.1088/1361-6595/ab5bf6

[39] J. Teunissen, U. Ebert. J. of Physics D: Applied Physics, 50 (47), 474001 (2017). doi.org/10.1088/1361-6463/aa8faf

[40] D.V. Beloplotov, V.F. Tarasenko, M.I. Lomaev, D.A. Sorokin. IEEE Trans. Plasma Sci., 47, 4521 (2019). DOI: $10.1109 /$ TPS.2019.2907998

[41] Ю.Д. Королев, Г.А. Месяц. Физика импульсного пробоя газов (Наука. Глав. ред. Физматлит, М., 1991). [Y.D. Korolev, G.A. Mesyats. Physics of pulsed breakdown in gases (URO-press, Ekaterinburg, 1998).]

[42] А.Н. Ткачев, С.И. Яковленко. Письма в ЖЭТФ, 77 (5), 264 (2003). [A.N. Tkachev, S.I. Yakovlenko. J. Exp. Theor. Phys. Lett. 77, 221 (2003).] doi.org/10.1134/1.1574835

[43] А.М. Бойченко, А.Н. Ткачев, С.И. Яковленко. Письма в ЖЭТФ, $78(11), 1223$ (2003). [A.M. Boichenko, A.N. Tkachev, S.I. Yakovlenko. J. Exp. Theor. Phys. Lett. 78, 709 (2003).]

[44] J.R. Dwyer, Z. Saleh, H.K. Rassoul, D. Concha, M. Rahman, V. Cooray, J. Jerauld, M.A. Uman, V.A. Rakov. J. Geophys. Res.: Atmos., 113, D23207 (2008). DOI: $10.1029 / 2008 J D 010315$

[45] J.R. Dwyer, D.M. Smith, S.A. Cummer. Space Sci. Rev. 173, 133 (2012). DOI: 10.1007/s11214-012-9894-0

[46] G.A. Mesyats. IEEE Trans. Plasma Sci., 23 (6), 879 (1995). DOI: $10.1109 / 27.476469$

[47] E. Kalered, N. Brenning, I. Pilch, L. Caillault, T. Minéa, L. Ojamäe. Phys. Plasmas, 24, 013702 (2017). doi.org/10.1063/1.4973443

[48] T.L. Chng, A. Brisset, P. Jeanney, S.M. Starikovskaia, I.V. Adamovich, P. Tardiveau. Plasma Sources Sci. Technol., 28 (9), 09LT02 (2019). doi.org/10.1088/1361-6595/ab3cfc

[49] A. Brisset, K. Gazeli, L. Magne, S. Pasquiers, P. Jeanney, E. Marode, P. Tardiveau. Plasma Sources Sci. Technol., 28 (5), 055016 (2019). doi.org/10.1088/1361-6595/ab1989

[50] Г.А. Аскарьян. Письма в ЖЭТФ, 1 (3), 44 (1965). [G.A. Askar'yan. Soviet J. Experimental Theor. Phys. Lett., 1, 97 (1965).

[51] L.P. Babich. High-energy phenomena in electric discharges in dense gases: Theory, experiment, and natural phenomena (Futurepast, Arlington, 2003).

[52] A. Kozyrev, V. Kozhevnikov, N. Semeniuk. In EPJ Web of Conferences. EDP Sciences, 167, 01005 (2018). doi.org/10.1051/epjconf/201816701005

[53] П.А. Бохан. УФН, 188 (12), 1361 (2018). [P.A. Bokhan. Physics-Uspekhi, 61 (12), 1241 (2018).] doi.org/10.3367/UFNe.2018.04.038362

[54] D. Levko, S. Yatom, V. Vekselman, J.Z. Gleizer, V.T. Gurovich, Y.E. Krasik. J. Appl. Phys., 111 (1), 013304 (2012). DOI: $10.1063 / 1.3676198$

[55] Е.Х. Бакшт, С.Я. Беломытцев, А.Г. Бураченко, В.В. Рыжов, В.Ф. Тарасенко, В.А. Шкляев. ЖТФ, 82 (7), 102 (2012). [E.K. Baksht, S.Y. Belomyttsev, A.G. Burachenko, V.V. Ryzhov, V.F. Tarasenko, V.A. Shklyaev. Tech. Phys., 57 (7), 998 (2012).] DOI: 10.1134/S1063784212070031 